healing satisfactorily follows this treatment much opacity of course remains. Among out-patients these infective ulcers are, perhaps, the least common, but they are said to occur with frequency among agricultural labourers, especially during harvest time.

In the foregoing remarks it will have been observed that I have more especially referred to two drugs-namely, chinosol and eserine-and about each of these I now propose to add some further details. With regard to chinosol, concerning the advantages of which I have been repeatedly asked, I believe it to be the best antiseptic agent which is now in the market, and the longer I use it the more I prefer it and the closer does it seem to me to fulfil the requirements of an ideal preparation of the kind. There are certain reasons for doubting whether in ophthalmic surgery the principles of antisepticism are followed in the present day to the extent which their importance demands, and it is probable that the want of precision in this regard is partly due to the fact that among the multiplicity of antiseptic agents there is none which has actually found general favour with ophthalmic surgeons. The perchloride of mercury, carbolic acid, boric acid, each has its drawbacks. Each is undoubtedly useful in its way, but neither the one nor the other has ever excited sufficient enthusiasm to cause thorough antiseptic principles to prevail in the domain of ophthalmology. The importance of this matter I believe to be such that $I$ hold sympathetic ophthalmia would be an impossible complication in eye surgery were a wound of the ciliary region to be treated from the first with every antiseptic precaution. In fulfilment of this belief I have treated within the past year several severe wounds in the dangerous zone, and in each case the eye made an excellent recovery: The results I attributed to the systematic antisepticism practised by means of chinosol. The chemical name of this drug is potassium. oxyquinoline sulphonate and one of its chief advantages is the potency of its germicidal action--a fact which has been incontestably proved by bacteriological investigation. In addition, it is freely soluble in water and thus is handy for use. Moreover, it is non-caustic, does not injure the skin of the hands, does not coagulate albumin, and is nonhygroscopic. Again, it is one of the most economical preparations in the market, for owing to its potency only weak solutions of the drug are necessary. Mixed with 1 in 20 of boric acid it forms an admirable antiseptic ointment. It might, perhaps, seem that in saying all this $\mathrm{I}$ am praising chinosol inordinately. The fact, however, is that a lengthened experience has taught me that it is an excellent preparation, possessing advantages which in time must commend it to every operating surgeon. With respect to eserine, there is some unanimity of opinion among the authors of modern text-books on ophthalmic surgery that the drug should be avoided in the treatment of corneal ulcers. But my experience, as will have been gathered, does not accord with that view. On the other hand, I believe that the feeling which prevails against eserine in this connexion has arisen in consequence of misconception regarding its use. The great point to remember is to use it in a weak solution. The evil repute of the drug has been mainly gained by employing solutions of greater strength than were necessary. Formerly eserine drops of the strength of four grains to an ounce of water used frequently to be prescribed, and then troublesome symptoms were nearly always induced. But it is seldom, if ever, necessary to resort to a higher strength than half-a-grain to an ounce solution, and for continuous instillation in cases of chronic glaucoma even a less strength than this may be satisfactorily employed. Experience has fully demonstrated that there are certain forms of ulceration of the cornea which atropine fails to benefit but which, on the other hand, readily yield to eserine. To define what these particular forms are is a question which has been repeatedly asked in my out-patient room and the answer may here be given as follows: all sloughing, infective and vascular ulcers are best treated with eserine as well as, of course, those situated at the corneal margin at which perforation is threatening. This may be regarded as a broad rule for guidance, but there remain other cases concerning which no rule can be expressed in words. I have in my mind those cases of simple ulcer in which atropine has been used and apparently failed. In many of such I have found eserine to act like a charm, the injection of the globe quickly clearing up in consequence and the ulcer rapidly beginning to show improvement. A typical case may here be mentioned. A short time ago a man wearing a shade was ler in to my out-patient room by his wife. The history was that he had been under treatment at an ophthalmic hospital for five weeks. There was a superficial central ulcer of each cornea with photophobia and some injection of the globes. The pupils were wiclely dilated from atropine. This seemed to be a case in which eserine was clearly indicated. Accordingly eserine drops ( $\frac{1}{2}$ gr. to an ounce of water) were ordered to be instilled twice a clay. At the end of the week when the patient again presented himself the improvement was very manifest. Two days after using the drops the patient discarded the shade and the photophobia had gone as well as the injection of the globes. Before the end of another week he had returned to his work. Undoubtedly in cases where photophobia is troublesome eserine is of service by contracting the pupil and cutting off light to the eye. while atropine increases the trouble by dilating the pupil, thus placing the patient with his damaged cornea in a helpless condition in the presence of light beyond a low degree. It is quite possible that the utility of eserine in the treatment of corneal ulcers is partly due to the fact that the drug reduces the tension of the globe. This is only a reasonable assumption when we recollect that atropine tends to raise the tension and that an eye in which increased tension has occurred is not one in which healthy nutrition is likely to be present. By lowering the tension, then, in these cases just the difference may be made in securing the establishment of those nutritional changes necessary for the complete repair of the ulcers.

\section{A CONTRIBUTION TO THE PATHOLOGY OF INFECTION BY THE PNEUMO-} COCCUS.

BY W. H. BRODIE, M.D. ABERD.,

W. G. ROGERS, M.D., M.S. LoND., $\triangle \mathrm{ND}$

E. T. E. HAMILTON, M.D., M.S.LOND., F.R.C.S.ENG., B.SC.

Ix 1894, in a paper read before the Transvaal Medical Society under the title of "Acute Specific Rhinitis," we communicated the result of an investigation we had made on an epidemic which had occurred amongst the Kaffirs. employed in one of the mines here. At least 100 cases. came under our notice. These were mostly of a mild form; many, however, were severe; and out of 15 deaths we were able to perform 9 post-mortem examinations. The salient features of the disease were purnlent discharge from. the nostrils and, in a large majority of the cases, pneumonia. Our special attention was first drawn to this epidemic by the sudden and unexpected deaths, unaccounted for by any premonitory constitutional disturbance or signs of sufficient local disease, even when pneumonia was present.

In order to lead up to our recent work a recapitulation of our previous inquiry is desirable. A summary of the post-mortem examinations is as follows. In 1 case we found purulent meningitis and pneumonia, with a large quantity of pus bathing the mucous membrane of the nasal cavities and filling the sinuses and ethmoidal cells. This Kaffir had worked seemingly well on the day of his death; he was quite conscious and could articulate up to within a few hours of his death. In 2 cases we found meningitis without pneumonia, with a small quantity of pus in the nasal regions and sinuses. One of these patients had worked on the day of his death; the other had only: complained of feeling ill for a day or tro. In 2 other cases there was pneumonia without meningitis and. a very large quantity of pus in the nasal sinuses and fossa. In 1 there was slight pneumonia without meningitis and no. where was there pus formation. This Kaffir had complained of pain in the head and loins for three days before he was seen. twelve hours before death he showed great constitutional disturbance. His temperature was $104^{\circ} \mathrm{F}$. and his pulse was. 130. He was sensible. Incessant romiting set in and be. gradually became comatose and there was incontinence of urine and fæces. We expected to find cerebral lesions, but they were absent. In one case we found neither meningitis nor pneumonia, and in this case again the constitutional disturbance was grave; the patient had lain unconscious for three days with incessant vomiting. In all the above 
necropsies we found one condition present - a livid, injected, swollen condition of pituitary membrane. One especially interesting post-mortem examination was made. This was on the emaciated corpse of a Basuto, aged about fortyfire years, who had been sent to hospital suffering from donble pneumonia. He had had purulent nasal discharge and injected and livid-coloured mucous membrane over the turbinate bones. The donble pneumonia was extensive, but the constitutional disturbance was slight in comparison. The temperature fell to normal on the eighth day. Sixteen dars after admission be had a severe attack of dysentery which lasted about a week. When discharged a month after admission he was well except that dulness persisted over the bases of the lungs. He died a month after leaving hospital ; the immediate cause of death was epistaxis. At the postmortem examination some recent pneumonia was found at the apices, also some old consolidation going on to gangrene, but the condition of the nasal cavities chiefly arrested attention. The whole of the diseased mucous membrane had exfoliated in the form of sloughs, leaving a pallid surface; the mucous membrane of the sinuses was pale and thin, and parts of the ethmoidal cells were denuded of micous membrane and periosteum. The apertures were remarkably patent. In one or two places the sloughs still adhered, with petechiæ in their neighbourhood. We sent 9 cases to hospital; all of the patients had red and turgid mucous membrane of the nose with more or less discharge and increased temperature; 6 had pneumonia with an abundant fine frothy expectoration-in one case four pints in twenty-four hours; 2 had no pneumonia. Jaundice was a marked feature in some of the cases and it was present in 2 at the post-mortem examinations, There was no evidence of disease of the nervous system in any of the hospital cases and they all recovered except the one case already alluded to. None of them had great constitutional disturbance, not even those with severe pneumonia. From a study of this epidemic we came to the conclusion that it was the result of a poison circulating in the blood, infecting first the nasal region and causing death by the toxæmic state which it produced, the meningitis and pneumonia being secondary and accidental-the result of the poison circulating in the blood.

Since the occurrence of that epidemic we have had many sporadic cases and have performed several necropsies, meeting the same characteristics, but there had been no serious outbreak until about six months ago, when the increased working of the mine demanded a large number of fresh hands. During the last four months about 800 raw Kaffirs came in batches into a new, commodious, and well-cared-for compound and among these new arrivals another epidemic occurred. They were nearly all of poor physique and came from the neighbourbood of Inhambane on the east coast, 100 miles north of Delagoa Bay, thence by train four days to Johannesburg, including a day's stoppage at the border to obtain passes. They suffered severely from sea-sickness and according to their report began to get ill with coughs on leaving the steamer. The winter had been exceedingly cold and they must have suffered severely from the low temperature, travelling insufficiently clad from the seacoast to this high altitude. In fact, the only protection each had against it was a cotton handkerchief. Like their fathers for generations, at home they did no work, living on wild vegetables, sugar-cane, plantains, and occasionally the flesh of wild pigs and a little milk. After a short but trying journey they arrived from a warm and comparatively moist sea climate to this high, dry, and cold one to work in a mine $1000 \mathrm{ft}$. below the surface, their diet consisting of an unlimited quantity of mealie meal varied with fresh meat, and a supply of lime-juice. Their powers of resisting disease must have been consider ably weakened by this sudden change in their habits, diet, and surroundings. A large majority on arrival were suffering from nasal catarrh and coughs, with pneumonia in one or two cases, but whether this was due to the specific organism infecting them before arrival or only acting as one factor in predisposing them to the attack here we have not yet been able to determine. During the months of June and July, the coldest months, the average number off work was about 100 . They appeared to be suffering from ordinary colds complicated in various cases with pneumonia, dys entery, diarrhcea, parotitis, and in two cases with meningeal symptoms. Out of the total number of sick 40 died, and we were able to obtain 17 post-mortem examinations
One batch of 93 emaciated Kaffirs arrived in the beginning of July, when the temperature, except for a few hours during the day, persisted below freezing point; a fortnight later only 16 were working and some of them were ailing. Altogether of this batch 8 died. Owing to our being now able to pursue our study into the bacteriology of the disease we have advanced a large step towards its elucidation, and although we were unable to make bacteriological examinations in the first epidemic a comparison of the clinical and pathological characteristics of the two outbreaks leaves no doubt that they were due to the same cause. The only differences were that in the first epidemic there were several cases of jaundice and only 1 with dysentery, and this only exemplifies what we wish to emphasise-i.e., how protean the manifestations of the disease are. At first sight it was natural to suppose that the various cases of pneumonia, dysentery, parotitis, diarrhoea, and spinal meningitis were distinct and independent illnesses brought on according to the idiosyncrasy of the individual by his being unable to withstand the new conditions of life. We performed post-mortem examinations on cases in which each of the above conditions was the immediate cause of death and the pathological and bacteriological examinations proved that we were dealing with only one disease. In our former paper we gave each case in detail, its history and clinical features, as far as we were able to observe them, and the post-mortem appearances, showing the charts with notes of the cases treated in the hospital. We think it superfluous, even if space allowed, to describe seriatim each of the numerous cases of the later epidemic. We shall therefore only enumerate the various symptoms.

The most prominent symptoms were nasal catarrh and coughs. The compound was sonorous with coughing. This was unaccompanied by signs of bronchitis or lobular pneumonia, but in a great many cases, even when the men were at work, it was associated with lobar pneumonia. Although all cases of nasal catarrb may not have been specific, yet as a diseased condition of the mucous membrane of the nose was found in all the necropsies it is fair to assume that the large majority were due to specific rhinitis. The same can be said of the pneumonias. In nearly all the necropsies pneumonia was found and proved to be of specific origin; there can therefore be little doubt that the majority, if not all, of the cases were due to the same cause. Indeed, to reason otherwise would mean that the disease we are considering is invariably fatal-that only the 40 dead were attacked by this specific organism. The mere presence of pneumonia was not a dangerous symptom. In the large majority of cases in which it was present it involved only a small part of the lung, generally at the bases, and the Kaffirs contentedly worked with lungs in this condition. In cases in which the lungs were extensively invaded recovery took place and only in one necropsy was the pneumonia found of sufficient extent to account for death. There was always coexisting pleurisy. Parotitis was a common symptom, but, like pneumonia, was not a guide to prognosis. Diarrhcea, not noted in the previous epidemic, was prevalent. It was not of a severe form, but it was of serious import, because in some of the cases the motions gradually assumed a dysenteric character and in all of the cases in which there was dysentery which came under our notice the patients died. In the great majority of the fatal cases dysentery was the immediate cause. A characteristic of this phase of the disease was that the evacuations towards the end of the illness became entirely hæmorrhagic, mostly darkcoloured, sometimes black, and simulating the motions found in purpura hæmorrhagica. When they were of this nature they were passed involuntarily. No treatment was of avail in lessening or modifying the discharge. The following case shows that when dysentery is the pronounced symptom there is a tendency to hæmorrhage in other organs.

A Kaffir had worked for two weeks after arrival without complaining of feeling ill; he then came into the mine hospital seemingly suffering from ordinary dysentery. A few days after admission dulness with tubular breathing developed at the bases of the lungs, but there was never a large area involved. He was never heard coughing or seen expectorating. The motions, at first of the usual dysenteric type, gradually became hæmorrhagic and twelve hours before death he was passing involuntarily large quantities of very dark-coloured blood. He had no signs of disease of the nervous system. At the post-mortem examination the mucous membrane of the nose was found to be less altered than usnal. Parts of the lung at the bases had red hepatisation. An abscess in 
the wall of the sphenoidal sinus, pus in other sinuses, and hæmorrhagic cerebral effusion were found. Considered as a symptom of the disease, cerebral meningitis could almost be excluded. We had only two cases with well-marked signs of meningeal mischief and in both spinal meningitis was found post mortem. This latency is remarkable when we think of the number of cases in which we found extensive purulent cerebral meningitis at the necropsies ; it was almost as often found as preumonia.

We suggested in our former paper that the brain of the uncivilised Kaffir may be less responsive to disease than that of the white man, but the lung mischief showed the same latency; we only knew of its presence in most cases because it was easily discoverable by physical examination. It has yet to be decided whether this latency is due to a peculiarity of the disease or of the individual. Before entering into the pathology and bacteriology we will draw attention to a case which illustrates the latency of the meningitis and how easy it is to miss the diagnosis of the disease.

A Kaffir was absent from work for two weeks suffering from painful swollen legs; he was only seen two days before death and then only perfunctorily examined. Scorbutus was suspected. He never appeared very ill and an hour before death he was seen sitting before the fire joining with animation in a conversation. He must have then methodically rolled himself in his blanket near the fire, for he was found dead in this condition. $\mathrm{He}$ had died quietly. At the post-mortem examination the femoral veins were full of thrombi. There were general purulent cerebral meningitis, slight pneumonia, and the usual condition of pituitary mucous membrane.

Another case illustrating the acutely toxic nature of the disease was that of a fine Kaffir who had only been in the compound a week. He had not been working. For two days before death he had complained to his friends of feeling sick, but on the day preceding his death he said he was better and announced his intention of going to work on the morrow. He was found dead in the middle of the night. The following is from the notes of the post-mortem examination made ten hours after the death. The face was swollen. There was emphysematous crepitation along the course of the large veins. There was a bloody nasal flux. The nasal mucous membrane was swollen and livid. There were from seven to eight ounces of hæmorrhagic pericardial effusion almost black. Red hepatisation was present at the upper part of the lower lobe of the left lung; there was no consolidation of the right lung. There was recent pleurisy on both sides. The spleen was enlarged and very soft. The muscles showed marked post-mortem staining; the blood was tarry throughout the body. There was neither jaundice nor meningitis. The bacteriological examination in the three cases cited showed that they were the result of acute general infection by the pneumococcus.

Morbid anatomy.-The 26 necropsies made in the course of our investigations were in all cases commenced by sagittal section of the skull. The thoracic and abdominal viscera were examined in every case, and the spinal cord in 5 cases. The morbid conditions observed were noted at the time of examination and the present account is a résumé of those notes. The necropsies were made as soon as possible after death and in many cases the bodies were still warm. Many of the examinations had to be conducted within the walls of a mine compound and it is of some psychological interest to record the fact that the Kaffirs viewed our operations with favour, pleased with the idea that we were seeking "strong medicines" to heal them in their sickness, which, indeed, was ultimately if not primarily true. Their own habit is in accordance with this conceit, for they are accustomed to lay under tribute to this end the lions or other large game which they succeed in killing in their native districts. They subsequently, however, resented our taking organs for preservation from emaciated subjects.

In all the cases after death we observed a greenish, thick, purulent discharge coming from the nostrils. In many cases emission of semen had occurred about the time of death. The nasal mucosa, including that of the adjacent sinuses, showed every stage of inflammation. We found oedema, hyperæmia with purple colouration, purulent infiltration, and ulceration. In more chronic cases there was atrophy to such an extent that the tissue was pallid and the various apertures leading from the nasal fossæ were abnormally patent. This appearance was specially marked at the orifices of the Eustachian tubes, the margins of which were in these instances remarkably sharp and pallid, being covered by the thinnest of mucous membranes. On the contrary, in other cases so great was the thickening that these openings were obscured by tumour.like masses. In many of the cases the bones forming the walls of the nasal fossæ were bare and necrotic. Sloughs were observed in the vault of the pharynx and on the upper turbinated bones. In one case the walls of the sphenoidal sinus were quite denuded of muco-periosteum. Indeed, this sinus in the majority of the cases showed serere changes, being generally filled with gelatinous, greenish pus, or exhibited a collection of pus between the periosteum and the bony wall. It was always gravely implicated in the meningitis cases. The same conditions were observed in the frontal sinus and in the other air spaces communicating with the nose. Within the skull cavity purulent infiltration of the arachnoid and pia mater extended backwards, usually from the optic groove, or in some cases from the crista galli to the foramen magnum from the base it spread over the conrexities to involve the mesial surfaces of the hemispheres. The pus was seen in the processes of the pia mater dipving into the sulci and followed the reflexion covering the choroid plexus of the lateral ventricle; the membrane in this position was thick with œdema. Over the cribriform plate of the ethmoid in some cases the meningitis was especially marked; also along the ophthalmic veins at their entry into the cavernous sinus. The cranial nerve-sheaths of the first eight pairs were often profoundly affected; sometimes the intracranial part of the optic nerve was obscured by the exudation. The undersurface of the pons was involved in some instances and in both of the spinal cases. The cerebellum nsually escaped. In one case referred to in the clinical part of this paper, we found hæmorrhagic effusion into the choroid plexus and velum interpositum; in the subarachncid cisterna between the posterior surface of medulla and cerebellum; also in the fourth rentricle and on the ventral surface of the pons round the basilar artery. In 1 case examined otitis media was associated with the rhinitis and meningitis. Spinal leptomeningitis was found in 2 cases out of 5 in which the cord was examined. In 12 cases out of 26 meningitis was. present. The exudation in some cases was jelly-like, homogeneous, and clear both in the spinal and cerebral meninges. In others, however, the inflammatory material present was fluid rather than jelly-like, opaque and granular rather than clear; and we surmised at the time that these latter characters might be dependent upon the presence of tine commoner pyogenic organisms in addition to that of the specific diplococeus.

The condition of the lungs and pleura varied; croupous pneumonia was present in the majority of the cases, but in a few the lungs were healthy. All stages of the pneumonic process were represented, from congestion to gangrene, and the extent of lung involved differed within very wide limits. Recent pleuritic lymph always accompanied the pneumonic consolidation. Effusion into the pleural cavity was often present but never exceeded a few ounces. In no case was endocarditis found. The pericardial sac contained excess of fluid in the majority of instances; the great vessels often showed excessive post-mortem staining. In one case cedema of both legs was noticed during life, and on examining the common femoral reins ante-mortem coagula were found The peritoneal cavity usually showed a considerable amount of fluid. Perihepatitis and perisplenitis were marked features in one case, in which, however, there was also extensive recent pleurisy on both sides accompanying red hepatisation; the spleen weighed $12 \mathrm{oz}$., and both it and the liver were firmly adherent to the diaphragm. The infestion was obviously general, for both cerebral and spinai meninges were involved. We examined the joints in some of the cases, always with a negative result. Double parotitis was observed once. In those cases in which dysenterio symptoms bad been present no gross change was seen on inspection of the intestine and the mesenteric vessels appeared to be healthy. A question of interest is the inquiry as to the path taken by the organism from the nose to the brain and other viscera. As a result of our examinations we think the most usual course to the interior of the skull is from the muco-periosteum of the sphenoirlal sinus to the sheaths of those nerves embedded in the wall of the cavernous sinus and thence by direct extension to the arachnoid and pia mater. In other cases undoubtedly the organism infects the meninges by extension along the nerves or vessels traversing the cribriform plate of the ethmoid ; for it has been shown that the nasal lymphatics are in com: munication with the subdural and subarachnoid spaces by 
means of perineural paths. Another possible route is by the Eustachion tube to the middle ear and petrous bone, thence by the serenth and eighth nerre sheaths.

The parotil glands are possibly infected from the mouth, though we think it more likely that they share the general infection wlien that is established. The lungs suffer in the same erent, but probably sometimes by direct extension along the respiratory tract. Tre arrived at this conclusion from the rarity of antecedent laryngitis, tracheitis, or bronchitis, from the general distribution of the specific organism throughout the body, and from the character of the pneumonia, which was lobar, basal, and fibrinous. We remember having seen some years ago in the post-mortem room, several cases of purulent meningitis in which a primary focus of suppuration was diligently sought for withont success. We remember one case in particular, that of a man who had twenty-four hours before admission first evidenced illness in change of conduct and sentiment. He died next day and on inspection suppurative meningitis was found withont any apparent source of infection. We are inclined to believe that a bacteriological examination of the pus in this case would have revealed a pneumococcal infection.

Pathology - Fifteen cases were subjected to a complete bacteriological examination. 1 . In every case cultivations were made on gelatin, agar agar, blood agar, and in broth. These were taken (a) from the pus in the nasal fossæ and in the nasal, frontal, splenoidal, ethmoidal and superior maxillary sinuses; (b) from the purulent exudate in the arachnoid, cerebral and spinal; $(c)$ from the fluid in the cerebral ventricles; $(d)$ from the pericardial, peritoneal, and pleuritic Aluids; $(e)$ from the heart blood and blood in the cranial venous sinuses; and $(f)$ from the lungs, kidneys, and spleen. In all cases we were careful to exclude the possibility of infecting our cultures by using the hot iron freely and subsequently making incisions with sterilised instruments. We were also fortunate in being able to conduct the bacteriological examinations very soon after death.

In our cultivation experiments we obtained the organism described below in pure culture from either the spleen, the pericardial fluid, or the heart blood of 7 cases. In some of the cases we obtained the organism in pure culture from all three sources named. In the remaining 8 cases we obtained no organisms of any kind in these (pericardial, heart blood, and spleen) tissues in our cultures (one tube inoculated from each). In 2 cases we obtained pure cultures of the organism from the cerebral exudate, in 5 others examined it was associated with streptococci and staphylococci. The characters of the organism thus obtained are as follows.

Morphology.-Microscopical examination showed the pure cultures to consist of lanceolate micrococci, nearly always united in pairs and tending in old cultures to grow into chains, rarely occurring as single cocci. In recent cultures, particularly on blood agar, the cocci are surrounded by a transparent halo-like capsule which is most obvious in coverslip preparations from the recent tissues and which disappears in cultures on any media. Since the organism most commonly occurs as such we have called it a diplococcus throughout this paper. It stains well with aniline colours and by Gram's method. The capsule is also well seen after double staining with methylene blue and eosine.

Biological characters. - The diplococcus grows in the presence of oxygen. It is not motile. We find that it grows best in media having a slightly alkaline reaction and that the capsule is retained longest when the organism is grown on blood agar prepared according to the method advocated by Dr. Washbourn and Dr. Eyre, of London. Of its growth on gelatin we would prefer to reserve our cpinion. Our experiments were made in the autumn and the room temperature very often varied $20^{\circ} \mathrm{F}$. in the twenty-four hours. Once or twice we thought we were getting a growth on gelatin but we were never satisfied on this point. In broth it grows well, rapidly producing a cloudiness throughout the medium. In this medium we found that the organism was still pathogenic for rabbits at the end of four days. Upon blood agar the diplococcus forms minute, almost colourless colonies at the end of from twenty-four to forty-eight hours. Numerous coverslip preparations were made from the fresh tissues in every case and from the same sources from which cultures were taken. Microscopical examination of these coverslips showed the presence of the diplococcus which we have described throughout the body. In 9 of the cases we found this organism and no others in the heart blood, the pericardial fluid, and the spleen. The preparations taken from the nasal fossw were, of course always very mixed, but even in these the diplococcus was in great excess. In the pus from neighbouring sinuses various organisms were found, but here next to the diplococcus the most numerous organisms were streptococci and staphylococci. In the purulent exuclate involving the arachnoid in 8 cases we found only the diplococcus: in the remainder streptococci and staphylococci were associated with it. From our microscopical examinations it seemed that the diplococcus first invaded the cerebral tissues, followed very speedily by the streptococci and staphylococci. In the two cases in which we examined the spinal arachnoid only the diplococcus was present. Preparations from the lung were always very mixed, but as before the diplococcus was in great excess. In the kidneys and the liver the diplococci were not as numerous as in the spleen.

Inoculation experiments.-Cultures and spleen juice injected into the peritoneal cavity of rabbits killed the animals in from twelve to forty-eight hours. After inoculation the temperature of the animal speedily rose to from $3^{\circ}$ to $5^{\circ} \mathrm{F}$. above normal and continued so until shortly before death when it usually fell. Post-mortem examination showed an cdematous infiltration of the cellular tissues, chiefly about the seat of inoculation, while small hæmorrhagic spots were common throughout the body, the whole appearances being exactly as we had seen them in Dr. Washbourn's laboratory at Guy's Hospital after inoculation with the pneumococcus of Fränkel. In the spleen, the heart blood, and the œdematous infiltrations in these animals we found the diplococcus in immense numbers and again obtained it in pure culture. We also injected a virulent blood agar culture into the subcutaneous tissue of the neck of a horse. Six hours later the animal appeared to be very ill. The temperature was $107^{\circ}$ and the respirations were from 50 to 60 . He refused his food and continued in this condition for five days, when the temperature fell, his appetite returned, and he slowly recovered after an abscess had formed at the seat of inoculation.

Conclusions. - From these observations and experiments we conclude :-1. That the diplococcus we have described is identical with the pneumococcus of Frïnkel but that probably it is of a more virulent type. 2. That the pneumococcus is the specific factor in the causation of cerebrospinal meningitis. 3. That in these cases the pneumococcus first affects the nasal mucous membrane and produces there a definite local lesion (rhinitis), and that all other subsequent pathological effects-infection of frontal and nasal sinuses, middle-ear suppuration, parotitis, pneumonia, meningitis, \&c.-are merely the result of the extension of the organism along various anatomical channels of infection-e.g., continuity of tissues, lymphatics, and blood-stream. The postmortem appearances of the nasal region which led us in our first observations on this disease, before we had made any bacteriological investigations, to name it acute specific rhinitis will serve to show how strongly we were convinced of its local origin. 4. That a pneumococcic septicæmia the result of a pneumococcic rhinitis, even when unattended by such secondary effects as pneumonia or meningitis, is sufficient to cause death. May not this explain the "fulminating attacks" of epidemic cerebrospinal meningitis of previous writers? It is interesting to compare the pathology of the disease under considera tion with that of infection by the bacillus tuberculosis. In the latter the presence of a definite bacillus in every case of attack, whether the field be lung or brain, proclaims the specific nature of the invasion. We think that the observations here recorded are simply further illustrations of the multiform effects produced in the body by the life activity of an equally deinite micro-organism and that further research will establish the contention that a general infection by the pneumococcus with its diverse lesions is often the complement of an unsuspected acute specific rhinitis.

In drawing this communication to a close we are conscious that there are many points which yet require attention and which we feel confident will throw further light on the apparent vagaries of this striking disease. We have been unable, for instance, to work out the history of the not infrequent presence in the cerebral meninges of streptococci and staphylococci, but from the fact that we have never found these organisms in the blood, the spleen, or the tissues we are inclined to the belief that they invade the brain only after the pneumococcus has prepared the wayan opinion which microscopical results appear to confirm Neither have we had time to trace by means of histological sections the course of the pneumococcus from the nose to the brain. We have as yet done no work on immunity, but we 
hope that the high virulence of this pneumococcus will facilitate the preparation of serum which may be efficient in cases of ordinary "pneumonia" as occurring in Europeans. in excuse for these many shortcomings we can only say that the present work was carried out in the spare time snatched from our private practices, and that we feel constrained to rpublish our results thus early in the hope that other observers will establish our chief contention that infection by the Dneumococcus is primarily a purely local phenomenon.

Johannesburg, South Africa.

THE USE OF FORMALIN LAMPS FOR THE DISINFECTION OF ROOMS (ALFORMANT LAMPS AND FORMOGENE RICHARD).

6 Y A. A. KANTHACK, M.A., M.D. LoND., F.R.C.S. ENG., F.R.C.P. LOND.,

EELLOW OF KIXG'S COLLEGE, CAMBRIDGE; PROFESSOR OF PATHOLOGY, CAMBRIDGE UNIVERSITI.

(From the Pathological Laboratory of the University of Cambridge.)

THE disinfection of rooms, furmiture, and bedding is a matter of great importance to all general practitioners and respecially to those who live in the country. It must be the desire of all to possess a method which is both simple and certain and, at the same time, not destructive in its action. Numerous papers have appeared recently which have occupied themselves with the question of the disinfecting power of formalin vapour. It is generally allowed that formalin vapour is a powerful disinfectant and it therefore promises to replace those gaseous substances which have hitherto been in use and of which some are useless and others injurious to man and the objects around. Lately simple and complicated forms of apparatus have been designed for the rapid and continued production of formalin vapour, and these by their manufacturers have been recommended as being unfailing in their disinfecting action. Writers abroad, however, are gradually recognising the dimitations of the formalin process and there is now an almost general consensus of opinion that formalin in the manner in which it is produced by almost all the different apparatus in use has only a limited power of penetration. There is no necessity for me to survey the extensive literature rapon this subject of disinfection of rooms by means of formalin vapours, since it may be found in almost any recent wolume of the Centralblatt für Bacteriologie and the Zeitschrift für Hyiene.

In this country three formalin lamps are in use, and having been frequently asked regarding their respective value I andertook a series of experiments in order to satisfy myself on this point, especially since others have already spoken with unveiled enthusiasm of these lamps. The Formalin Hygienic Company supply two simple apparatus-viz., the Alformant Lamps A and B; and the Germicide Disinfecting Association a somewhat more complicated lamp, the Formogène Richard Lamp. In this paper I propose to describe a number of experiments which were made under laboratory conditions with the purpose of independently testing the efficiency of these three tlamps, which all profess to accomplish similar results. By means of laboratory experiments, however, we can only decide under what conditions formalin vapours are likely to act; the final verdict must be pronounced when an apparatus has been tested under practical conditions. The principle of the Alformant lamps depends upon the conversion of solid paraform into active formalin gas by means of heat in the presence of water and carbonic acid. Alformant Lamp $\overline{\mathrm{B}}$ is a small, toy-like apparatus, and it is stated in the directions for use that one tablet of paraform is required for the ordinary sterilisation of 1000 cubic feet of air and ten tablets are required for thorough disinfection and certain destruction of all pathogenic germs in 1000 cubic feet of space, whether these be scattered about superficially or hidden in bedding, stuffed seats, books, \&c. Alformant Lamp A is merely a larger model; otherwise it is said to act in exactly the same manner as Alformant Lamp B. In the Formogène Lamp the formalin is obtained by means cf the incomplete combustion of strong wood spirit. It is unnecessary for me to describe the mechanism of the lamps and I shall at once proceed to state my results.

1. Alformant Lamp $B$ (small pattern).--Two sets of experiments were made and in each case 25 tablets were burnt. A room was used, the windows, fireplace, and other openings of which were carefully closed and sealed, so that little or no formalin could or did escape. It has recently been stated that such stringent precautions are not necessary, but it seemed adrisable under the circumstances to prevent any loss of the formalin vapour. The measurement of the room was $18 \mathrm{ft}$. by $12 \mathrm{ft}$. by $9 \mathrm{ft}$. - that is, its capacity was well under 2500 cubic feet, so that, according to the instructions, 25 tablets should have amply sufficed for complete disinfection. As test objects I used small strips of linen which bad been dipped (1) in 24 hours old broth cultures of anthrax bacilli; (2) in 24 hours old broth cultures of typhoid bacilli; (3) in 24 hours old broth cultures of staphylococcus pyogenes aureus; (4) in sewage effuent; and (5) in an old typhoid stool. These were subsequently dried over sulphuric acid at $38^{\circ} \mathrm{C}$. The dust of the room also served as a test object and animals were placed in the room during the experiment. The strips were distributed in the room at different places, some openly exposed on the benches and on the floor, others wrapped up in ordinary dusters. After 24 hours the room was opened and the strips at once dropped into broth, and the latter was then incubated at $38^{\circ} \mathrm{C}$. The results of these 2 experiments are given in tabular form.

Experiment $I$. (Alformant Lamp $B$ ).

\begin{tabular}{|c|c|c|}
\hline Test material. & $\begin{array}{l}\text { Exposed on } \\
\text { table or floor. }\end{array}$ & $\begin{array}{l}\text { Wrapped in } \\
\text { dusters. }\end{array}$ \\
\hline Linen strips dipped in sewage $\ldots \quad \ldots$ & + & - \\
\hline 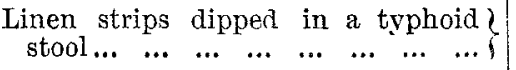 & \pm & - \\
\hline 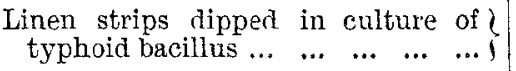 & + & - \\
\hline $\begin{array}{c}\text { Linen strips dipped in cultures of } \\
\text { anthrax bacillus } \ldots \\
\ldots\end{array}$ & + & - \\
\hline $\left.\begin{array}{l}\text { Linen strips dipped in culture of } \\
\text { staphylococcus pyogenes aureus ... }\end{array}\right\}$ & + & - \\
\hline $\left.\begin{array}{r}27 \text { hours old culture of anthrax } \\
\text { bacillus on agar-agar } \ldots \quad \ldots \quad \ldots\end{array}\right\}$ & - & - \\
\hline $\begin{array}{lllllllll}\text { Dust } & \ldots & \ldots & \ldots & \ldots & \ldots & \ldots & \ldots & \ldots\end{array}$ & \pm & $\ldots$ \\
\hline
\end{tabular}

$+=$ completely sterilised $;-=$ not sterilised; ani $\underline{t}=$ sometimes sterilised, at other times not sterilised.

We learn from this experiment that only the most superficially exposed material was disinfected and that the formalin failed to penetrate even through thin dusters.

A second experiment was arranged in exactly the same manner, 25 tablets being used as before. In this case all the linen strips, whether superficially exposed or not, had escaped the action of the formalin, so that this experiment was altogether negative, although all possible precautions had been taken.

Experiment II. (Alformant Lamp B).

\begin{tabular}{|c|c|c|}
\hline Test material. & $\begin{array}{l}\text { Exposed on } \\
\text { table or floor. }\end{array}$ & $\begin{array}{l}\text { Wrapped in } \\
\text { dusters. }\end{array}$ \\
\hline Linen strips dipped in sewage $\ldots \quad \ldots$ & - & - \\
\hline Linen strips dipped in typhoid stool & - & - \\
\hline $\left.\begin{array}{c}\text { Linen strips dipped in } 24 \text { hours old } \\
\text { broth culture of typhoid bacillus... }\end{array}\right\}$ & - & - \\
\hline $\left.\begin{array}{c}\text { Linen strips dipped in } 24 \text { hours old } \\
\text { broth culture of anthrax bacillus ... }\end{array}\right\}$ & - & - \\
\hline $\begin{array}{l}\text { Linen strips dipped in } 24 \text { hours old } \\
\text { broth culture of } \\
\text { pyogenes aureus ... } \\
\text { py }\end{array}$ & - & - \\
\hline $\begin{array}{c}24 \text { hours old agar-agar culture of } \\
\text { anthrax bacillus } \ldots \\
y^{\prime}\end{array}$ & - & - \\
\hline $\begin{array}{lllllllll}\text { Dust } & \ldots & \ldots & \ldots & \ldots & \ldots & \ldots & \ldots & \ldots\end{array}$ & - & $\ldots$ \\
\hline
\end{tabular}

In this experiment disinfection failed completely.

It is evident, especially when we also consider Experiment I., that Alformant Lamp B is uncertain in its action and therefore untrustwortby. Hence this lamp, however 